\title{
Radio interferometric tests of general relativity
}

\author{
E. B. Fomalont ${ }^{1}$ and S. Kopeikin ${ }^{2}$ \\ ${ }^{1}$ National Radio Astronomy Observatory, \\ 520 Edgemont Road, Charlottesville, VA 22903, USA \\ email: efomalon@nrao.edu \\ ${ }^{2}$ Dept. of Physics and Astronomy, \\ University of Missouri, Columbia, MO 65211, USA \\ email: kopeikins@missouri.edu
}

\begin{abstract}
Since VLBI techniques produce a microarcsecond positional accuracy of celestial objects, tests of GR using radio sources as probes of a gravitational field have been made. We present the results from two recent tests using the VLBA: in 2005, the measurement of the classical solar deflection; and in 2002, the measurement of the retarded gravitational deflection associated with Jupiter. The deflection experiment measured $\gamma$ to an accuracy of $3 \times 10^{-4}$; the Jupiter experiment measured the retarded term to $20 \%$ accuracy. The controversy over the interpretation of the retarded term is summarized.
\end{abstract}

Keywords. gravitation, techniques: interferometric, astrometry

\section{Introduction}

The theory of general relativity (GR) describes the interaction of matter and light with a gravitational field; hence, any accurate measurement of this interaction is a test of GR. The simplest test was the GR prediction of the angular deflection of starlight passing near the limb of the sun, first performed in 1916 during a solar eclipse. The results of other experiments, most using radio light rather than star light, agreed with the GR prediction to $<0.1 \%$ accuracy. Departures of $\gamma$ from unity are expected at the $10^{-6}$ level, and more accurate deflection observations will continue.

Other properties of gravity can be measured with different experiments. For example, the perihelion shift of Mercury is a measure of the non-linearity of the GR. The second measurement described in this paper, that of the retarded deflection of light caused by the motion of the gravitating body, obtained results in agreement with GR, but its interpretation with the property of gravity that is constrained by this experiment is controversial.

\section{The 2005 VLBA Deflection Experiment}

We believed that a new, well-designed, VLBA experiment could significantly improve upon the accuracy of previous solar radio deflection experiments for two reasons. With a stable electronic system, sensitive receivers and accurate astrometric/tropospheric modeling, the relative position of sources separated by a few degrees in the sky were now being routinely measured by the VLBA with about $20 \mu$ as accuracy. Secondly, the VLBA was now operating routinely at $43 \mathrm{GHz}$ where solar coronal effects are relatively small. With a typical troposphere coherence time of $1 \mathrm{~min}$ (maximum integration time per observation) and the compactness of most quasars, several groups of quasars within a five degree 


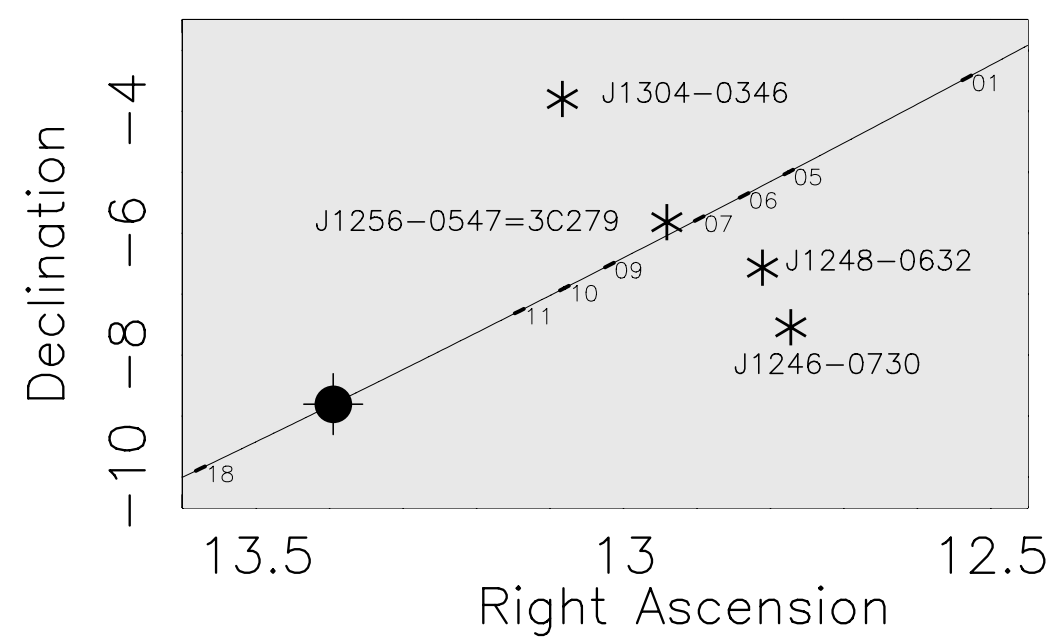

Figure 1. Source configuration and observation dates for the 2005 October deflection experiment:

region near the ecliptic were easily detectable. The source configuration that we selected for the October experiment is shown in Fig. 1.

We observed on 8 days, each for a period of 5 hours. On October 1 and 18, the relative gravitational and coronal bending was less than $2 \mu$ as so the undeflected relative positions for the sources could be determined. Also, any significant structural changes of the quasars over the experimental period could be ascertained (none were seen). Significant relative gravitational bending was obtained on the other observing days. The observations on each day were identical. Each source was observed for $45 \mathrm{sec}$ with a switching time of about $25 \mathrm{sec}$, in the sequence: 3C 279, J1304, 3C 279, J1256, 3C 279, J1258, 3C 279 , J1304, etc. As long as the phase between subsequent 3C 279 observations could be connected, this observing scheme accurately determined the source relative positions.

In order to determine the coronal refraction at $43 \mathrm{GHz}$, we time-shared the observations on each day among 43, 23, and $15 \mathrm{GHz}$. This was done by using the source observing sequence noted above, but switching frequencies every 25 minutes. In this manner we determined the relative position of the sources at any one frequency in each 25-min block to an accuracy of $\sim 100 \mu$ sec. By comparing the source positions as a function of frequency, we determined any significant frequency dependent position change. However, when a source was closer than about $2.2^{\circ}$ of the sun, the coronal refraction vibrated the source position at $43 \mathrm{GHz}$ more than $50 \mu$ sec over a time scale of a few seconds, making the phase tracking impossible to follow. Hence, all data were phase unstable for October 7 and several sources could not be used on October 5, 6 and 9. An additional $10 \%$ of the data were lost for some VLBA telescopes during periods of poor weather.

As a rough guide to the experiment sensitivity, at $43 \mathrm{GHz}$ the typical accuracy for the relative position between two sources over one five hour period was about 0.07 mas. With a relative gravitational deflection of 100 mas for most observing days, we obtained a deflection accuracy of 1 part in 1400 (0.0007) for each source-pair and day. When all source-pairs and are averaged at $43 \mathrm{GHz}$, we obtain $(\gamma-1)=-0.00070 \pm 0.00040$ (rms error). If we include the $23 \mathrm{GHz}$ and $15 \mathrm{GHz}$ data to remove the small coronal bending, we obtain $(\gamma-1)=-0.00006 \pm 0.00027$, our best estimate.

We believe that we can increase the experiment accuracy by a factor two to three. First, a group of sources in May when the sun is at a more northern declination will improve 
the astrometric quality of the data. Second, the most accurate results are obtained when the sources are between $3^{\circ}$ to $5^{\circ}$ from the sun. Third, the 2005 experiment scheduled too much time with sources too near the sun and not enough time at these intermediate solar separations. Finally, most of the observation time should be made at $43 \mathrm{GHz}$, with perhaps $25 \%$ of the time at $23 \mathrm{GHz}$ to remove the significant coronal bending.

\section{The 2002 Jupiter Deflection Experiment}

On September 8, 2002, Jupiter passed within $3.7^{\prime}$ of the quasar J0842+1835. Such a close passage of Jupiter with a bright quasar (0.4 Jy, among the brightest 1000 sources) occurs on average once every 20 years. A similar encounter in 1988 with a different bright quasar was observed, and the gravitational deflection of about 1 mas was detected by Treuhaft \& Lobe (1991). Our goal was to measure not only the radial deflection, but the retarded component as well. The relevant parameters at closest approach are shown in Fig. 2. The maximum radial deflection was $1190 \mu$ as and occurred at 16:30 UT on September 8. Because of the motion of Jupiter, there is a retarded deflection component of $51 \mu$ as opposite to the direction of motion of Jupiter, and it is within reach with the VLBA.

The details of the experiment and the results have been published by Fomalont \& Kopeikin (2003). The VLBA observed at $8.4 \mathrm{GHz}$ and observations switched between $\mathrm{J} 0842+1835$ with another quasar about $0.8^{\circ}$ east J0839+1802, and with $\mathrm{J} 0854+2006$, about $3.4^{\circ}$ to the west. A complete cycle took about 5 minutes. Five observing days, each 7 hours long, were made on September 4, 7, 8, 9, 12. Since the retarded term was significant only on September 8, the other four days were used to measure the undeflected position of the sources and to determine realistic errors.

These three quasars were positioned nearly linearly in the sky; hence, the appropriate combination of the measured phases for J0839 and J0854 not only removed the temporal troposphere and ionosphere refraction changes, but also the effect quasi-stationary phase gradients in the sky among the three sources. These systematic phase gradients are caused by many small astrometric effects (antenna location offsets, earth-orientation modeling errors), as well as troposphere and ionospheric structure from two to twenty degrees in the sky. The use of two calibrators rather than one reduced the residual position uncertainty per day from about $25 \mu$ as to about $10 \mu$ as (Fomalont (2003)).

Both the radial and retarded deflections were easily detected. Analysis of the radial deflection gave $\gamma=1.01 \pm 0.03$. The GR prediction of the retarded deflection varied between 41 and $51 \mu$ as on September 8, and the ratio of the measured retarded deflection to the GR prediction (assuming that the velocity associated with the retardation is the speed of light) was $0.98 \pm 0.19$.

The experiment confirms the GR prediction for the retarded deflection at the $20 \%$ level. A useful question is: what property of gravity is constrained by this experiment? Our interpretation is that the retardation is a measure of the propagation speed of gravity, and is related to a gravito-magnetic field associated with currents (motion) of matter (Kopeikin \& Fomalont (2007)).

A summary of other interpretations has been compiled by Will (2008). The basic disagreement is whether the speed of propagation of light or gravity (c) can be manifested in $\left(\mathrm{v}_{j} / \mathrm{c}\right)$ terms or only in $\left(\mathrm{v}_{j} / \mathrm{c}\right)^{2}$ terms, where $\mathrm{v}_{j}$ is the velocity relevant to the object. More specifically, Asada (2002) claims that the speed of light was determined. Will (2003) believes that the PPN parameter $\alpha_{1}$ was measured, albeit poorly. Carlip (2004) finds the distinction between the speed of gravity and the speed of light somewhat ill-posed. Stuart (2004) believes that the experiment measured nothing useful. 


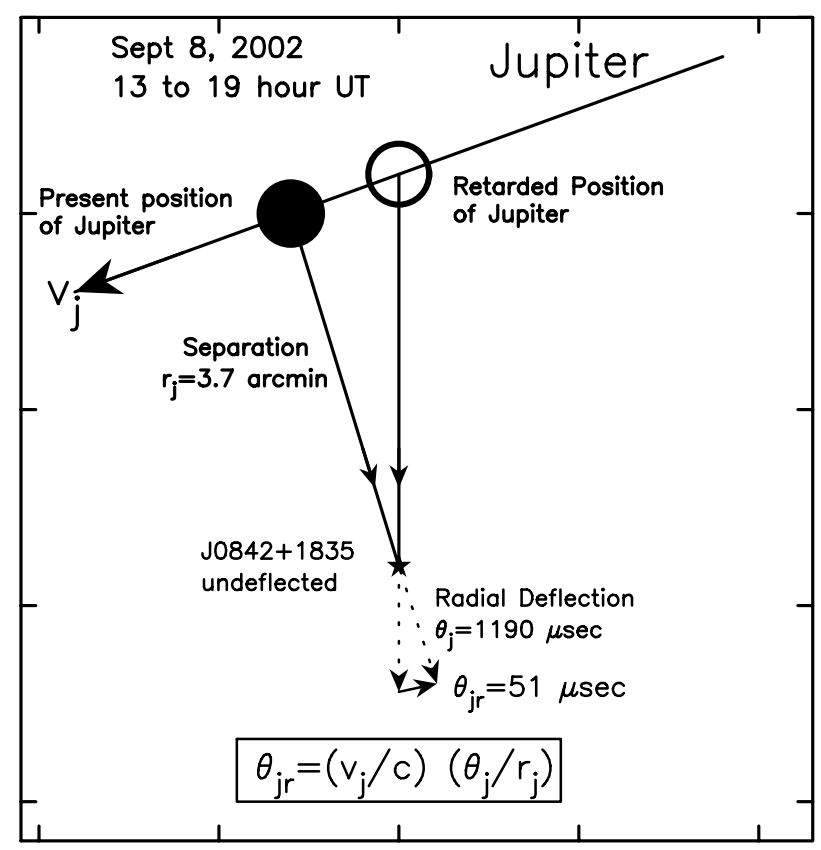

Figure 2. The Retarded Deflection of the Jovian Experiment in 2002: The line shows the path of Jupiter during the Sept 8 observations, with closest approach at 16:30 hr UT. The relevant deflection angles are shown.

\section{Summary}

The two experiments demonstrate that the micro-arcsecond positional accuracy of the VLBA produces significant tests of GR. The goal of the tests was to measure important parameters and their limits, and to foster discussion concerning the generalization of GR and its interaction with light and mass.

The National Radio Astronomy Observatory is a facility of the National Science Foundation operated under cooperative agreement by Associated Universities, Inc.

\section{References}

Asada, H. 2002, ApJ, 574, L69

Carlip, S. 2004, Class. Quant. Grav., 21, 3803 (arXiv:gr-qc/0403060v3)

Fomalont, E. B., 2003, Future Directions in High Resolution Astronomy, Ed. J. D.Romney and M. J. Reid, Socorro, NM, p55

Fomalont, E. B. \& Kopeikin, S. M. 2003, ApJ, 598, 704

Kopeikin, S. M. \& Fomalont, E. B. 2007, Gen. Rel. and Grav., 39, 1583 (arXiv:gr-qcd/0510077v4)

Stuart, S. 2004, Int. J. Mod. Phys., D13, 1753.

Treuhaft, R. N. \& Lowe, S. T. 1991, AJ, 102, 1879

Will, C. M., 2003, ApJ, 590, 683

Will, C. M., 2008, http://wugrav.wustl.edu/people/CMW/SpeedofGravity.html 